\title{
Tight focusing of radially polarized beams modulated by a fractal conical lens
}

\author{
Zhirong Liu ${ }^{1,2}$, Kelin Huang', Xun Wang ${ }^{1}$ and P H Jones ${ }^{2}$ \\ ${ }^{1}$ Department of Applied Physics, East China Jiaotong University, Nanchang 330013 \\ People's Republic of China \\ ${ }^{2}$ Department of Physics and Astronomy, University College London, Gower Street, \\ London WC1E 6BT, United Kingdom \\ E-mail: liuzhirong_2003@126.com
}

\begin{abstract}
A novel high numerical aperture (NA) focusing system with a fractal conical lens (FCL) is proposed, and tight focusing of radially polarized beams through the proposed optical system is investigated theoretically and numerically. The influence of several relevant factors, including the FCL's stage $S$, objective lens' NA, and truncation parameter $\beta$, on the targeted beam's focusing characteristics in the focal region is discussed in detail. It is found that, when a FCL with $S \geqslant 0$ is employed, position of the major focal point would shift from the geometric focal point, and the focused intensity distributions cannot maintain symmetrical about the focus any more, although they present different profiles for various truncation parameters $\beta_{0}$. When $S$ $\geqslant 2$, multiple focal points can be generated, i.e., a single major focus and a series of subsidiary foci surrounding it along the optical axis, which form a focal region. These unique focusing characteristics with a FCL are remarkably different from that of without a FCL. The fascinating findings here may be taken advantage of when using radially polarized beams in exploiting new-type optical tweezers and making use of a FCL.
\end{abstract}

Keywords: tight focusing, polarization, laser beam shaping

\section{Introduction}

In recent years, tight focusing of radially and azimuthally polarized light beams through a high numerical aperture (NA) objective lens has attracted intensive 
attention and extensive investigation, because of their fascinating features and wide potential applications [1-10]. It is found that, when radial polarizations are highly focused, a very strong longitudinal electric field component will emerge in the focal region, which forms an extremely tight focal spot. It is known that a tight focal spot is of great help to improve the resolution of microscopy [11, 12], enhance laser cutting ability in material processing [13], and can also be applied to improve the performance of optical tweezers due to the reduction of scattering $[10,14,15]$. Tight focusing of the electromagnetic field with various polarizations have been investigated, such as the linearly polarized, circularly polarized, radially polarized, azimuthally polarized, and hybridly polarized beams, as well as vortex beams [1-7]. It is also revealed in the vicinity of the focus that, for linear incident polarization, the generated longitudinal polarized component is not rotationally symmetric, which causes an asymmetric deformation of the focal spot [16, 17]; for radial polarization input, it generates a strong longitudinal electric field component in the focal zone [11, 18]; In contrast, the azimuthal incident polarization produces a strong magnetic field on the optical axis [19], meanwhile the electric field is purely transverse and null at the center [2]. When the hybrid polarized vector beams are highly focused, the focal shape may change from an elliptical spot to a ring focus with increasing the radial index, and meanwhile, the radial-variant spin angular momentum (SAM) of hybrid polarized vector beams is shown to be converted into radial-variant orbital angular momentum (OAM) [20]. One most recent report indicates that by dressing spatially variant polarization optical beams (e.g., azimuthally or radially polarized ones) with a vortex, one can generate at the focal plane subwavelength structures rotating with the optical frequency [21].

As a more general optical device than a conical lens (CL), the fractal conical lens (FCL) has its radial phase distribution following the Cantor function [22, 23]. Since its first demonstration by Monsoriu in 2006, research and applications on a FCL has attracted considerable interest due to the particular self-similarity characteristics under monochromatic illumination [22-25]. In the present work, we propose a novel high numerical aperture (NA) focusing system, in which a fractal conical lens (FCL) is 
employed, and investigate the tight focusing of radially polarized beams through the proposed optical system. This paper is structured as follows. In section 2, the theory model about the tight focusing of radially polarized beams modulated by a FCL is presented, and the main focusing formula are derived. In section 3, in terms of the formula obtained above, the influence of several relevant factors, including the FCL's stage $S$, objective lens' NA, and truncation parameter $\beta_{0}$, on the targeted beam's focusing characteristics in the focal region is numerically simulated and analyzed in detail. Finally, the main findings obtained are summarized in section 4.

\section{Theory Model}

As a new type of cylindrically symmetric diffractive lens, a fractal conical lens (FCL) has its radial phase profile designed from any given Cantor set (CS). As an example, figure 1(a) illustrates the construction of a regular triadic CS. The first step consists in defining a straight-line segment of unit length called the initiator (stage $S=0$ ). Next, at stage $S=1$, the generator of the set is constructed by dividing the segment into three equal parts of $1 / 3$ and removing the central one. Then the procedure is continued at the subsequent stages $S=2,3 \cdots$. It is easy to find that, in general, at stage $S$ there are $2^{S}$ segments of length $3^{-S}$ and $2^{S}-1$ disjoint gaps located at intervals $\left[p_{S, l}, q_{S, l}\right]$, with $l=1, \cdots, 2^{S}-1$. For instance, $S=3$, the triadic CS presents seven gaps at $[1 / 27,2 / 27]$, [3/27, 6/27], [7/27, 8/27], [9/27, 18/27], [19/27, 20/27], [21/27, 24/27], and [25/27, 26/7]. For clarity, the three first stages CS are depicted in figure 1(a). It should be noted that, a similar procedure could be followed for CS other than triadic.

Based on the fractal structure, the Cantor function $F_{S}(x)$ is defined in the domain $[0,1]$ as $[22,23]$

$$
F_{S}(x)=\left\{\begin{array}{ll}
\frac{l}{2^{S}} & \text { if } p_{S, l} \leq x \leq q_{S, l}, \\
\frac{1}{2^{S}} \frac{x-q_{S, l}}{p_{S, l+1}-q_{S, l}}+\frac{l}{2^{S}} & \text { if } q_{S, l} \leq x \leq p_{S, l+1}
\end{array},\right.
$$

where $S$ is the stage of the Cantor function, and $l$ is the number of disjoint gaps intervals $\left[p_{S, l}, q_{S, l}\right]$ that the function has. Here $q$ and $p$ denote the start and end points for each segment of the Cantor function, respectively, and $F_{S}(0)=0$ and $F_{S}(1)=1$. For example, when $S=3$, on the intervals, the constant values of $F_{3}(x)$ are $1 / 8,2 / 8,3 / 8,4 / 8$, 
$5 / 8,6 / 8$, and 7/8, respectively (see figures $1(\mathrm{a})$ and $1(\mathrm{~b})$ ). In between these intervals the continuous function increases linearly, as plotted in figure 1(b).

The FCL is a rotational symmetric pupil whose phase profile is designed from the Cantor function of a given stage $S$. Then, the phase of a FCL of stage $S$ is given by

$$
q(\varsigma)=q_{F C L}(\varsigma, S)=\exp \left[-\mathrm{i} 2^{S+1} \pi F_{S}(\varsigma)\right]
$$

where

$$
\varsigma=r / R
$$

is the radial variable $r$ normalized by the pupil radius $R$. Then, the surface-relief profile of the FCL can be expressed from the relation [26]

$$
h_{F C L}(r)=\bmod _{2 \pi}\left[-2^{s+1} \pi F_{S}\left(\frac{r}{R}\right)\right] \frac{\lambda}{2 \pi(n-1)},
$$

where $\bmod _{2 \pi}[\phi(r)]$ denotes the phase function $\phi(r)$ modulo $2 \pi, n$ represents the refractive index of the optical material used for constructing the lens, and $\lambda$ is the wavelength of the light.

Figure 1(c) illustrates the profile of a triadic FCL generated by equation (4) in the case $S=3$. One can find that there exist eight phase peaks in the domain $[0,1]$ along the radial direction, and the phase profile changes linearly versus the normalized radial coordinate $r / R$.

When illuminated by a monochromic light beam, the FCL's phase transmittance can be expressed as

$$
T(\theta)=\exp \left[-\mathrm{i} 2^{S+1} \pi F_{S}\left(\frac{\sin \theta}{\sin \alpha}\right)\right],
$$

where $\alpha=\sin ^{-1}\left(N A / n_{1}\right)$ denotes the maximum convergence angle determined by NA and $n_{1}$. Here NA is the objective lens' numerical aperture, and $n_{1}$ is the refractive index in image space.

In this work, we investigate the intensity distribution of highly focused radially polarized beams modulated by a FCL. In order to visualize the focusing procession, figure 2 illustrates the geometry of the tight focusing system. According to the vectorial Debye theory, when a radial polarized beam is focused by a high NA focusing system combined with a FCL, the radial and longitudinal components of the 
electric field in the focal region, respectively, have the following form [2, 27]

$$
\begin{aligned}
& E_{r}(\rho, z)=A \int_{0}^{\alpha} \cos ^{1 / 2} \theta \sin (2 \theta) l_{0}(\theta) T(\theta) J_{1}(k \rho \sin \theta) e^{i k \cos \theta} d \theta, \\
& E_{z}(\rho, z)=2 i A \int_{0}^{\alpha} \cos ^{1 / 2} \theta \sin ^{2} \theta l_{0}(\theta) T(\theta) J_{0}(k \rho \sin \theta) e^{i k \cos \theta} d \theta,
\end{aligned}
$$

where $A=\pi f \sqrt{n_{1}} / \lambda$ denotes a constant with $f, n_{1}$ and $\lambda$ being the optical focal length, refractive index of the medium, and optical length in vacuum, respectively. $l_{0}(\theta)$ represents the relative amplitude of the field in the pupil of the lens assumed to be a function of $\theta$ only. $\quad k=2 \pi n_{1} / \lambda$ denotes the wave number in image space, $J_{0}$ and $J_{1}$ represent the first kind of Bessel functions of orders 0 and 1, respectively. In addition, $T(\theta)$ denotes transmittance function of the FCL shown in figure 1(c), which will be used to modulate the radially-polarized incident beam.

In the following analysis, let us assume that the incident radial-polarization has a Laguerre-Gaussian profile [28]

$$
l_{0}(\theta)=E_{0} \beta_{0} \frac{\sin \theta}{\sin \alpha} \exp \left[-\left(\beta_{0} \frac{\sin \theta}{\sin \alpha}\right)^{2}\right] L_{p}^{1}\left[2\left(\beta_{0} \frac{\sin \theta}{\sin \alpha}\right)^{2}\right],
$$

where

$$
E_{0}=\sqrt{4 \beta_{0}^{2} P_{0} /\left[(p+1) \pi w_{0}^{2}\right]}
$$

is a constant related to the incident beam power $P_{0}$, and $L_{p}^{1}(\cdot)$ denotes the generalized Laguerre polynomial with $p+1$ rings. $w_{0}=f \sin \alpha$ is the beam width, and $\beta_{0}$ is a truncation parameter, which is defined as the ratio of the pupil radius $R$ to the incident beam radius $w_{0}$ in front of the focusing lens. Note that $\beta_{0}$ should be greater than 1 because some intensity distribution of the incident beam would be blocked by the pupil if $\beta_{0}<1$.

Therefore, the total intensity distribution of light beams in the focal region can be expressed as

$$
I(\rho, z)=\left|E_{r}(\rho, z)\right|^{2}+\left|E_{z}(\rho, z)\right|^{2} .
$$

In terms of Eqs. (5)-(9), evolution of the optical intensity distribution of highly focused radially-polarized beams modulated by a FCL can be examined in detail. 


\section{Numerical Results and Analysis}

Figure 3 shows the evolution of the total intensity distributions (2D left column, and $1 \mathrm{D}$ right column, below is the same) of tight focused radially polarized beams without a FCL for different truncation parameters $\beta_{0}$. It is clearly seen from figure 3 that, in the geometric focal region, the focused intensity presents a variety of distributions versus $\beta_{0}$. When $\beta_{0}=1.26$ the focused intensity distribution forms a dark area surrounded by the intense light field, which is known as optical cage, and meanwhile two symmetrical bright spots are located on the beam axis either side of the dark region (see figures $3(\mathrm{a} 1)$ and (a2)). When $\beta_{0}=1.64$ the intensity distribution emerges a transitional profile (see figures 3(b1) and (b2)). While the focused intensity generates a sharp peak-centered distribution when $\beta_{0}=2.02$ (see figure $3(\mathrm{~d} 1)$ and $(\mathrm{d} 2)$ ). Here, it is also noted that, without a FCL, the evolution of tight focused intensity distribution of radially polarized beams in the focal area is approximately symmetrical about the focal point, although it may produces a variety of profiles for various $\beta_{0}$.

Figure 4 depicts the evolution of the total intensity distributions of tight focused radially polarized beams modulated by a FCL in the case $S=0$ for different truncation parameters $\beta_{0}$. Comparing figure 4 with figure 3 , it is obviously noted that in this case none of the focused intensity distributions produces a dark-centered profile in the focal area any more. Clearly, they all form a sharp peak-centered configuration, and become asymmetrical about the focal point, even though they may present different profiles for various truncation parameters $\beta_{0}$.

When FCL's stage $S=1$ shown in figure 5 , it can be found that the width of intensity distribution becomes wider, and evolution of the total intensity distributions becomes more complex. It is also noticeable that the position of the major focal point shifts from the geometric focus in the presence of a FCL stage $S=1$.

Further increase FCL's stage to $S=2$, one can notice from figure 6 that, besides a major focal point, several subsidiary focal points arise along the axis direction, producing a focus region with unique fractal envelopes.

When $S=3$, as expected, the evolution of the total intensity distributions, shown in figure 7, exhibits a single major focus and a series of subsidiary focal points 
surrounding it, which generate a focus region. Of great interest is that, when $\beta_{0}=1.35$, in the geometric focal region, it produces a almost dark-hollow distribution. Increasing $\beta_{0}$ to 1.78 , the intensity in the focus is not null, and more subsidiary focal points emerge. Further increasing $\beta_{0}$ to 2.21 , then it forms a main peak profile in the geometric focus and a series of subsidiary focal points surrounding the main focus.

In order to investigate the influence of objective lens' numerical aperture (NA) on the focused radially polarized beams' intensity distribution, figure 8 depicts the evolution of on-axis intensity distributions for several NA. It is clearly seen from figure 8 that the maximum intensity enhances when increases NA, whether modulated by a FCL or not. Comparing figures $8(\mathrm{a})$ and $8(\mathrm{~b})$, one can also note that when NA is changed, without a FCL the focused intensity maintains a Gaussian-like distribution when $\beta_{0}=2.02$ (see figure 7(a)), while with a FCL $S=3$ the focused intensity distributions present a variety of profiles, although still maintain center-peaked configuration when $\beta_{0}=2.21$ (see figure $8(b)$ ).

Figure 9 illustrates the influence of truncation parameters $\beta_{0}$ on the tight focused radially polarized beams intensity distribution. It is obvious from figure 9 that the maximum intensity enhances when increase $\beta_{0}$ whether having a FCL or not, although the intensity distribution may change form dark-centered to peak-centered configurations for some different $\beta_{0}$.

\section{Conclusion}

In this work, we have proposed a novel high numerical aperture (NA) system with a fractal conical lens (FCL), and have investigated the tight focusing of radially polarized beams through the proposed optical system. Also, we have discussed the influence of several factors, mainly including the FCL's stage $S$, objective lens' NA, and truncation parameter $\beta_{0}$, on the targeted beam's focusing characteristics in the focal area. Results show that, when a FCL with $S \geqslant 0$ is employed, the major focal point would shift its position from the geometric focal point, and the focused intensity distributions can no longer maintain symmetrical about the focus, although they present different profiles for various truncation parameters $\beta_{0}$. When $S \geqslant 2$, multiple 
focal points can be generated, i.e., a single major focus and a series of subsidiary foci surrounding it, which form a focus region. These unique focusing characteristics with a FCL are remarkably different from that without a FCL. Owing to these intriguing findings, the novel high numerical aperture system, proposed here, may find applications in exploiting new-type optical tweezers and making use of a FCL.

\section{Acknowledgements}

This work was supported by the National Natural Science Foundation of China (Grant No. 11547002), the Jiangxi Provincial Natural Science Foundation of China (Grant No. 20161BAB201031), the Scientific Project of Jiangxi Education Department of China (Grant Nos. GJJ150531 and GJJ160467), and China Scholarship Council (Grant No. 201508360027).

\section{References}

[1] Dorn R, Quabis S, and Leuchs G 2003 Sharper focus for a radially polarized light beam Phys. Rev. Lett. 91233901

[2] Youngworth K S and Brown T G 2000 Focusing of high numerical aperture cylindrical vector beams Opt. Express 777-87

[3] Zhan Q and Leger J R 2002 Focus shaping using cylindrical vector beams Opt. Express $10324-331$

[4] Zhan Q 2006 Properties of circularly polarized vortex beams Opt. Lett. 31 $867-869$

[5] Yuan G H, Wei S B and Yuan X C 2011 Nondiffracting transversally polarized beam Opt. Lett. 36 3479-3481

[6] Hua L, Chen B, Chen Z and Pu J 2011 Tight focusing of partially coherent, partially polarized vortex beams J. Opt. 13075702

[7] $\mathrm{Hu} \mathrm{K}$, Chen Z and Pu J 2012 Generation of super-length optical needle by focusing hybridly polarized vector beams through a dielectric interface Opt. Lett. 37 3303-3305

[8] Chen Z, Hua L and Pu J 2012 Tight focusing of light beams: effect of polarization, phase and coherence Prog. Opt. 57 219-260 
[9] Khonina S N and Golub I 2016 Time behavior of focused vector beams J. Opt. Soc. Am. A 33 1948-1954

[10] Liu Z and Jones P H 2016 Optical manipulation using highly focused alternate radially and azimuthally polarized beams modulated by a devil's lens $J$. Opt. Soc. Am. A 33 2501-2508

[11] Quabis S, Dorn R, Eberler M, Glockl O and Leuchs G 2000 Focusing light to a tighter spot Opt. Commun. 179 1-7

[12] Dorn R, Quabis S and Leuchs G 2003 Sharper focus for a radially polarized light beam Phys. Rev. Lett. 91, 233901

[13] Nesterov A V and Niziev V G 2000 Laser beams with axially symmetric polarization J. Phys. D 33 1817-1822

[14] Kawauchi H, Yonezawa K, Kozawa Y and Sato S 2007 Calculation of optical trapping forces on a dielectric sphere in the ray optics regime produced by a radially polarized laser beam Opt. Lett. 32 1839-1841

[15] Nieminen T A, Heckenberg N R and Rubinsztein-Dunlop H 2008 Forces in optical tweezers with radially and azimuthally polarized trapping beams Opt. Lett. 33 122-124

[16] Sheppard C J R 2001 High-aperture beams J. Opt. Soc. Am. A 18 1579-1587

[17] Dorn R, Quabis S and Leuchs G 2003 The focus of light-linear polarization breaks the rotational symmetry of the focal spot J. Mod. Opt. 50 1917-1926

[18] Scully M O and Zubairy M S 1991 Simple laser accelerator: optics and particle dynamics Phys. Rev. A 44 2656-2663

[19] Zurita-Sa'nchez J R and Novotny L 2002 Multipolar interband absorption in a semiconductor quantum dot. II. Magnetic dipole enhancement J. Opt. Soc. Am. B $192722-2726$

[20] $\mathrm{Hu} \mathrm{K}$, Chen Z and Pu J 2012 Tight focusing properties of hybridly polarized vector beams J. Opt. Soc. Am. A 29 1099-1104

[21] Khonina S N and Golub I 2016 Ultrafast rotating dipole or propeller-shaped patterns: subwavelength shaping of a beam of light on a femtosecond time scale Opt. Lett. 41 1605-1607 
[22] Chalice D R 1991 A characterization of the Cantor function Amer. Math. Monthly $98255-258$

[23] Monsoriu J A, Furlan W D, Andrés P and Lancis J 2006 Fractal conical lenses Opt. Express 14 9077-9082

[24] Furlan W D, Saavedra G and Monsoriu J A 2007 White-light imaging with fractal zone plates Opt. Lett. 32 2109-2111

[25] Cheng S, Zhang X, Ma W and Tao S 2016 Fractal zone plate beam based optical tweezers Sci. Rep. 634492

[26] Han Y, Hazra L N and Delisle C A 1995 Exact surface-relief profile of a kinoform lens from its phase function J. Opt. Soc. Am. A 12 524-529

[27] Richards B and Wolf E 1959 Electromagnetic Diffraction in Optical Systems. 2. Structure of the Image Field in an Aplanatic System Proc. Royal Soc. London A. $253358-379$

[28] Quabis S, Dorn R, Eberler M, Glöckl O and Leuchs G 2001 The focus of light-theoretical calculation and experimental tomographic reconstruction Appl. Phys. B: Lasers and Optics 72 109-113 


\section{FIGURE CAPTIONS}

Figure 1. (Color online) (a) Illustration of the generation of the triadic Cantor set, starting from the initiator, $S=0$, to $S=3$; (b) the Cantor function $F_{S}(x)$ for $S=3$; (c) the phase of a triadic FCL for $\Phi_{s}=2^{s+1} \pi F_{s}(\varsigma) \quad$ with $S=3$ and ${ }_{\varsigma}=r / R$.

Figure 2. Schematics of highly focused radially polarized beams modulated by a FCL.

Figure 3. (Color online) Evolution of the total intensity distributions (2D left column, and 1D right column) of tight focused radially polarized beams without a FCL for different truncation parameters: (a1), (a2) $\beta_{0}=1.26$; (b1), (b2) $\beta_{0}=1.64$; (c1), (c2) $\beta_{0}=2.02$. The beam power in each transverse plane is normalized to $P_{0}=1 \mathrm{~W}$.

Figure 4. (Color online) Evolution of the total intensity distributions (2D left column, and 1D right column) of tight focused radially polarized beams modulated by a FCL in the case $S=0$ for different truncation parameter: (a1), (a2) $\beta_{0}=1.04$; (b1), (b2) $\beta_{0}=1.43$; (c1), (c2) $\beta_{0}=1.81$. The beam power in each transverse plane is normalized to $P_{0}=1 \mathrm{~W}$.

Figure 5. (Color online) Evolution of the total intensity distributions (2D left column, and 1D right column) of tight focusing radially polarized beams modulated by a FCL in the case $S=1$ for different truncation parameter: (a1), (a2) $\beta_{0}=2.04$; (b1), (b2) $\beta_{0}=2.59$; (c1), (c2) $\beta_{0}=3.14$. The beam power in each transverse plane is normalized to $P_{0}=1 \mathrm{~W}$.

Figure 6. (Color online) Evolution of the total intensity distributions (2D left column, and 1D right column) of tight focusing radially polarized beams modulated by a FCL in the case $S=2$ for different truncation parameter: (a1), (a2) $\beta_{0}=1.42$; (b1), (b2) $\beta_{0}=1.92$; (c1), (c2) $\beta_{0}=2.43$. The beam power in each transverse plane is normalized to $P_{0}=1 \mathrm{~W}$.

Figure 7. (Color online) Evolution of the total intensity distributions (2D left column, and 1D right column) of tight focusing radially polarized beams modulated by a FCL in the case $S=3$ for different truncation parameter: (a1), (a2) $\beta_{0}=1.35$; (b1), (b2) $\beta_{0}=1.78$; (c1), (c2) $\beta_{0}=2.21$. The beam power in each transverse plane is normalized to $P_{0}=1 \mathrm{~W}$.

Figure 8. (Color online) Evolution of on-axis intensity distribution of tight focused radially polarized beams without a FCL (a), and with a FCL (b) for several NA. The beam power in each transverse plane is normalized to $P_{0}=1 \mathrm{~W}$.

Figure 9. (Color online) Evolution of on-axis intensity distribution of tight focused radially polarized beams without a FCL (a), and with a FCL (b) for several $\beta_{0}$. The beam power in each transverse plane is normalized to $P_{0}=1 \mathrm{~W}$. 
(a)

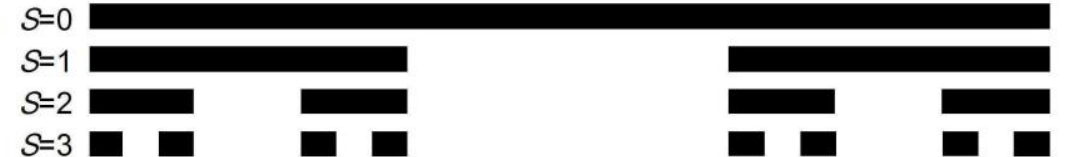

(b)
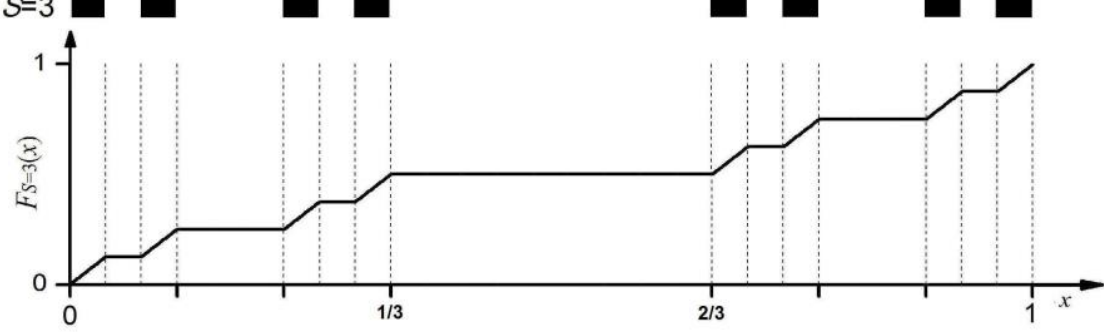

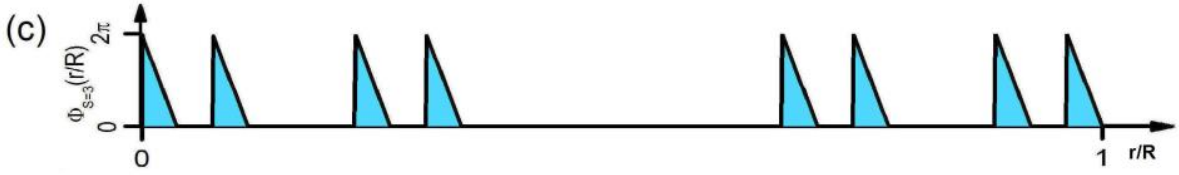

Figure 1. 


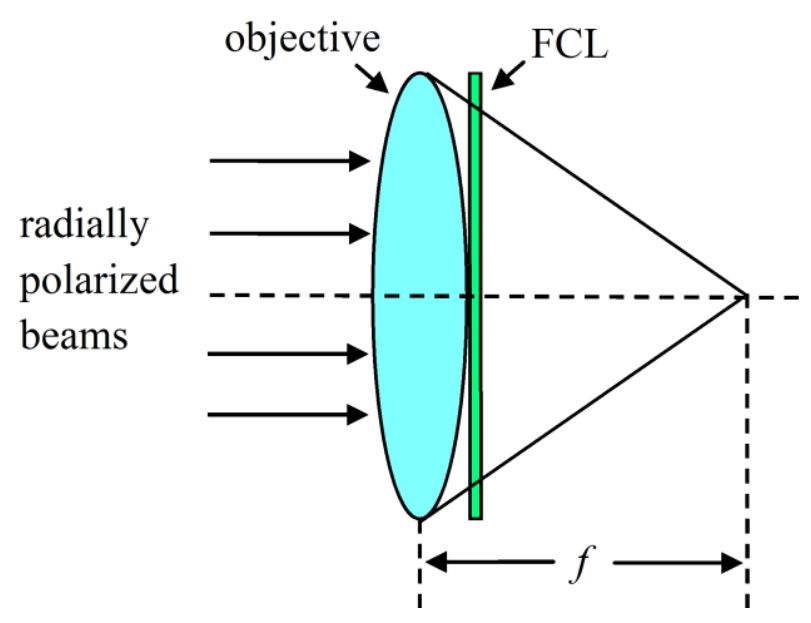

Figure 2. 


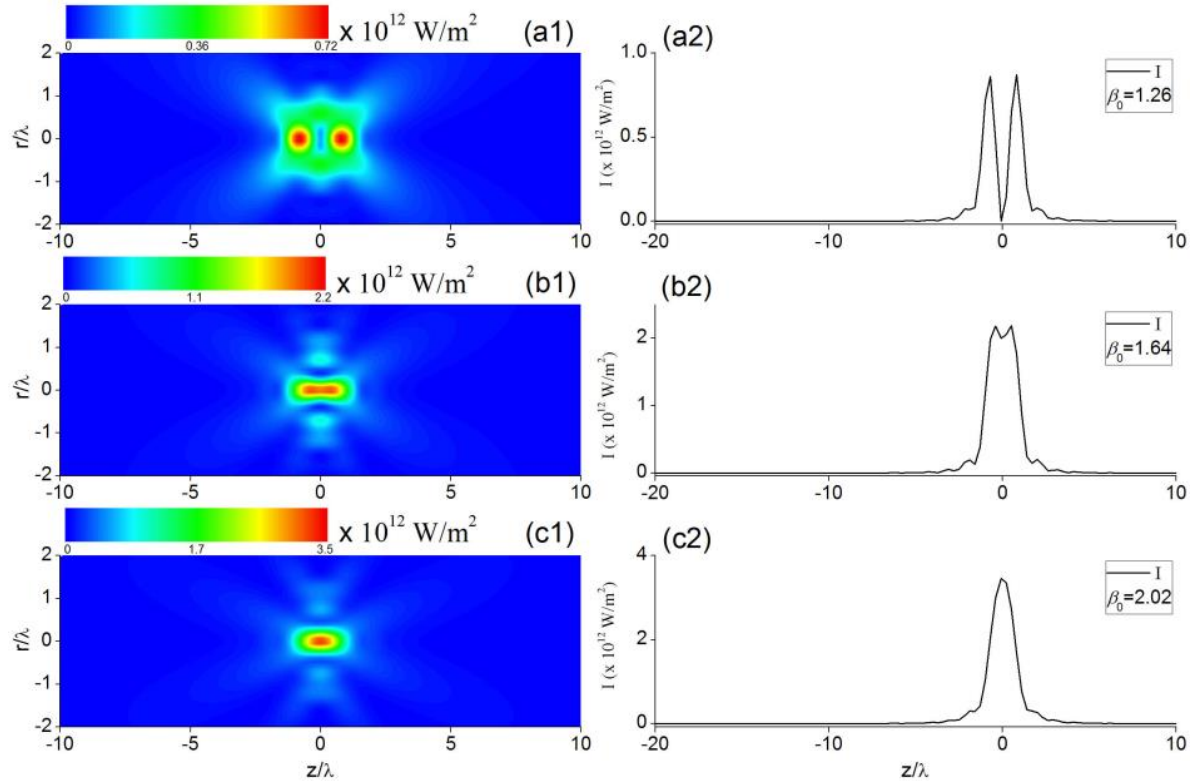

Figure 3. 


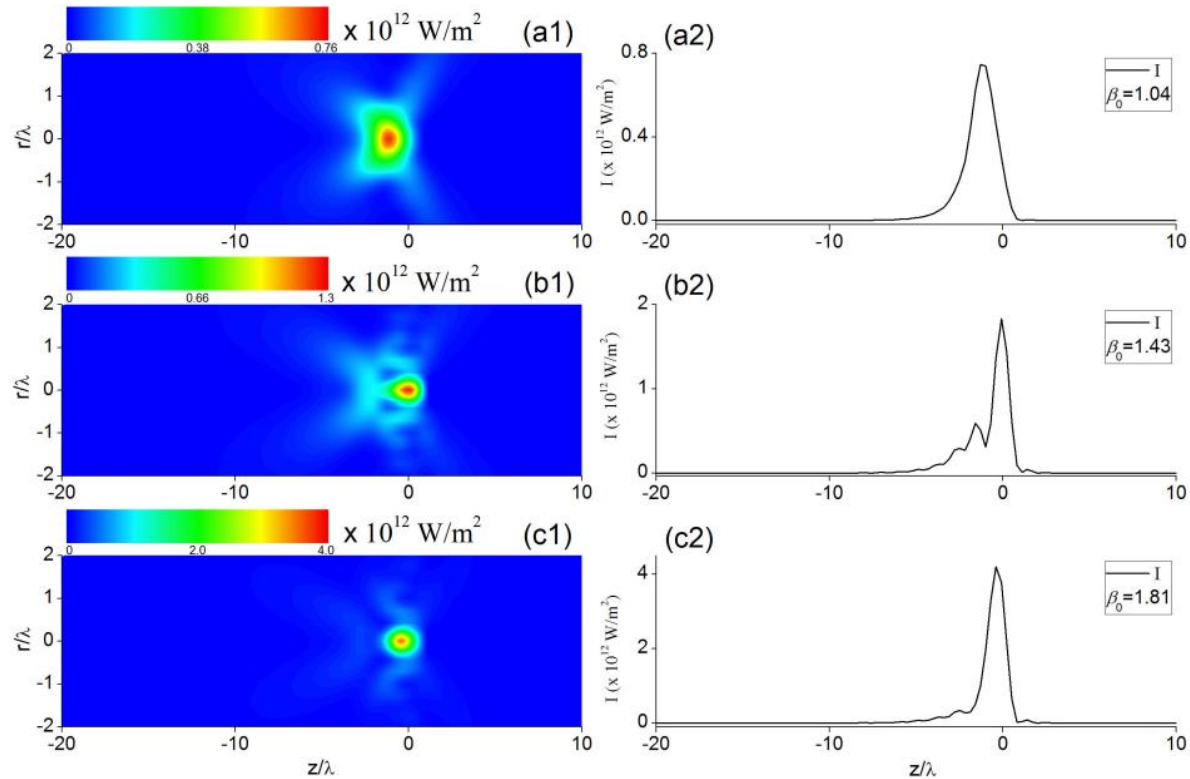

Figure 4. 


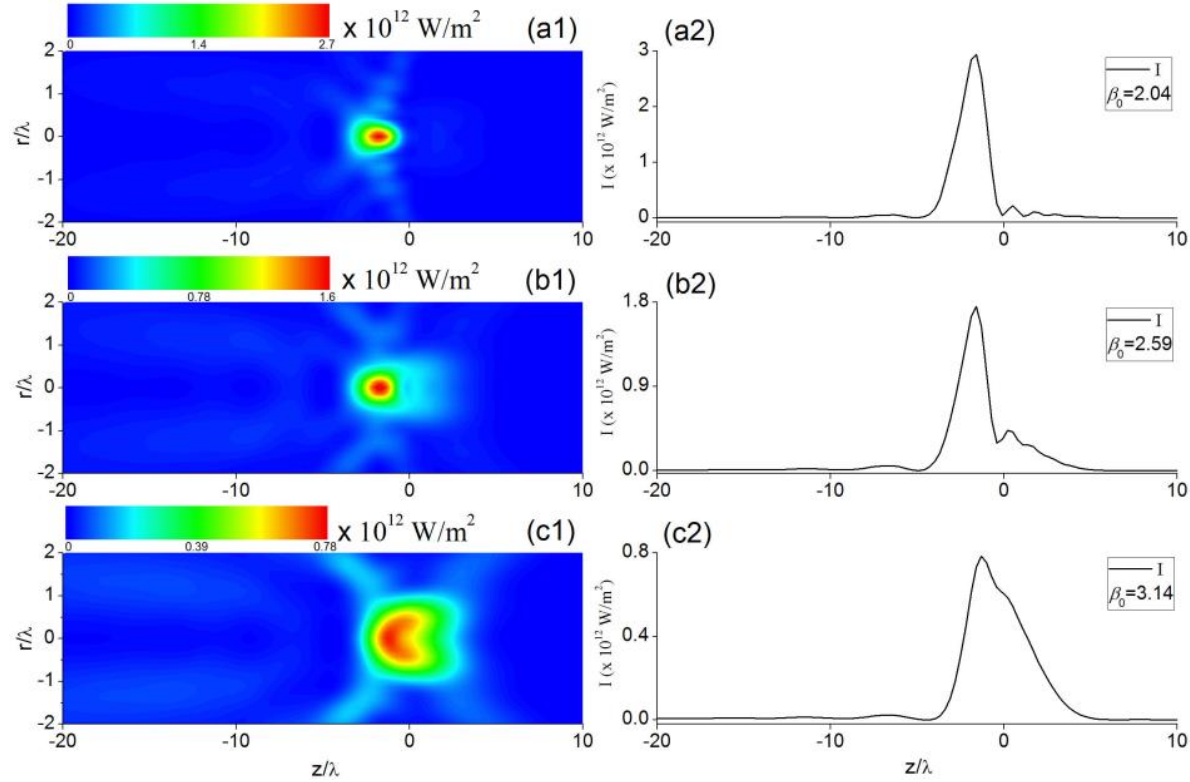

Figure 5. 


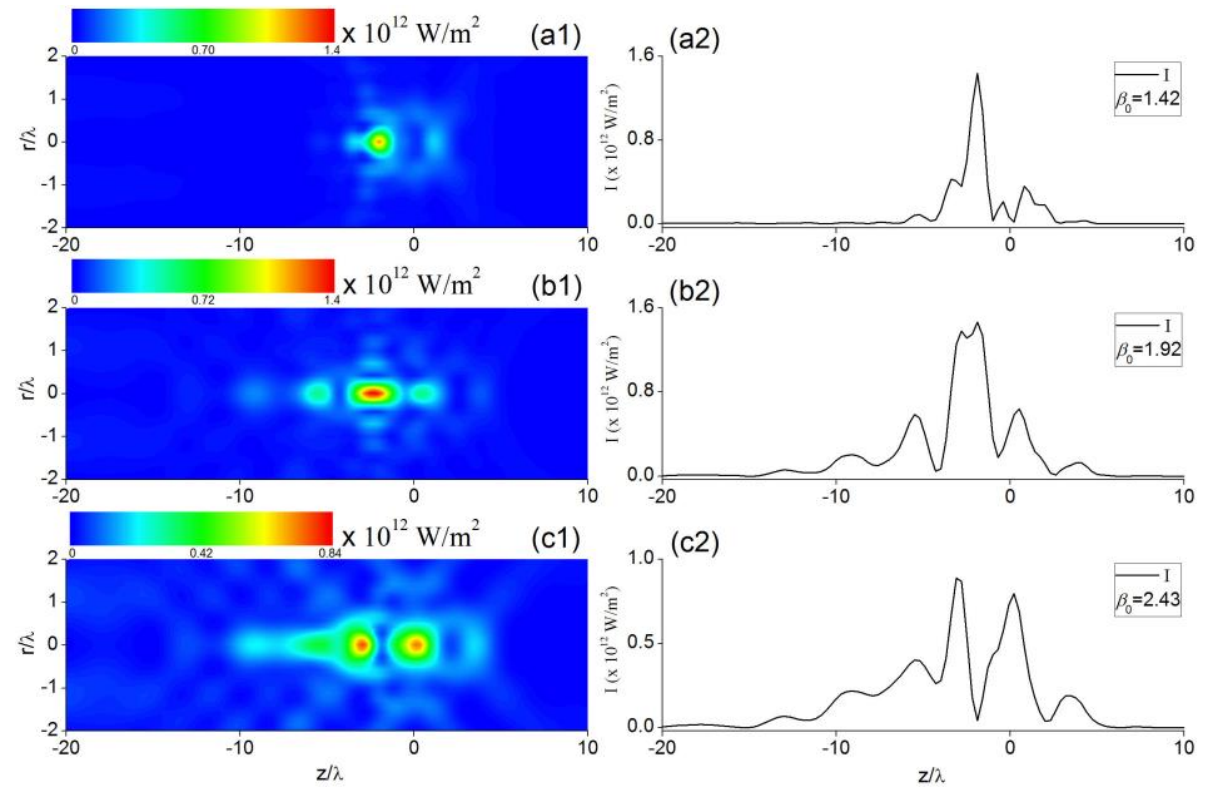

Figure 6. 


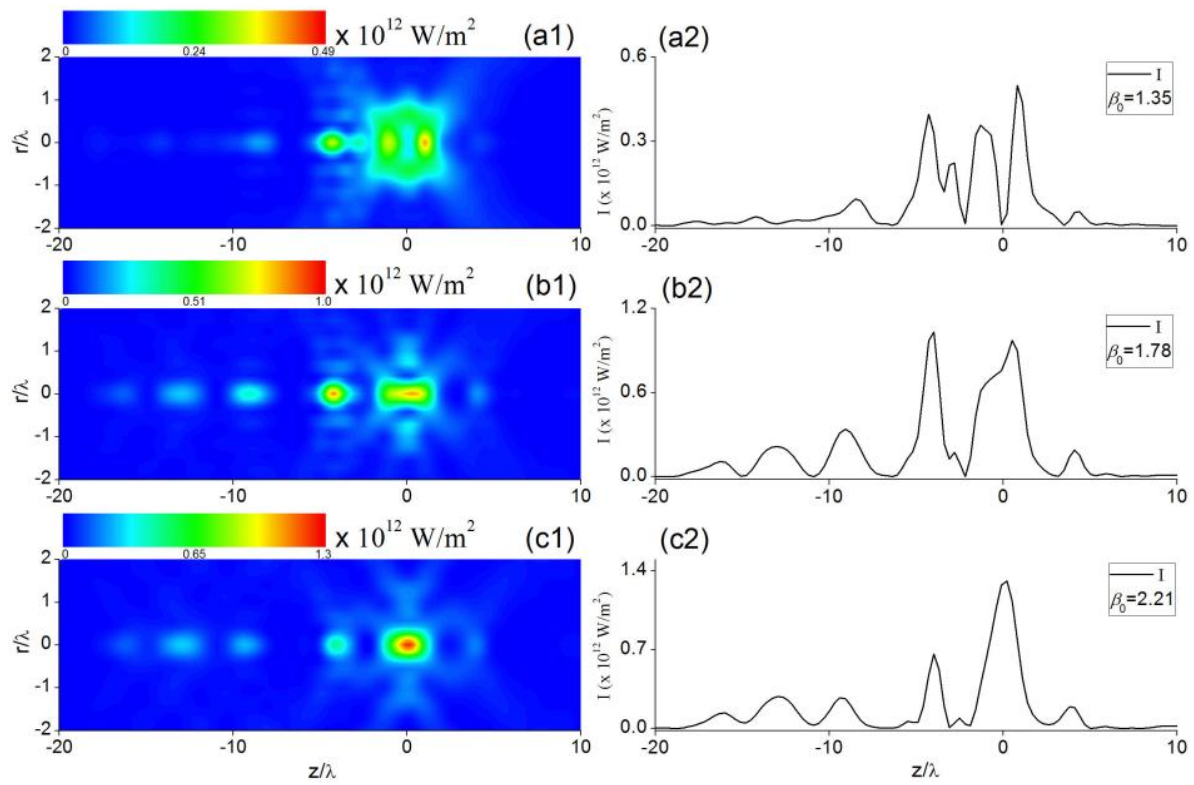

Figure 7. 

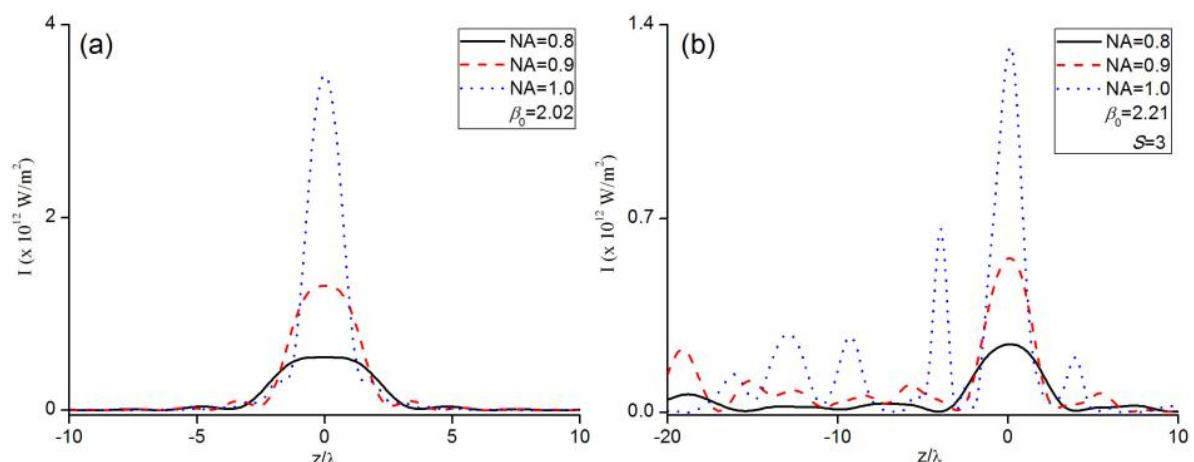

Figure 8. 

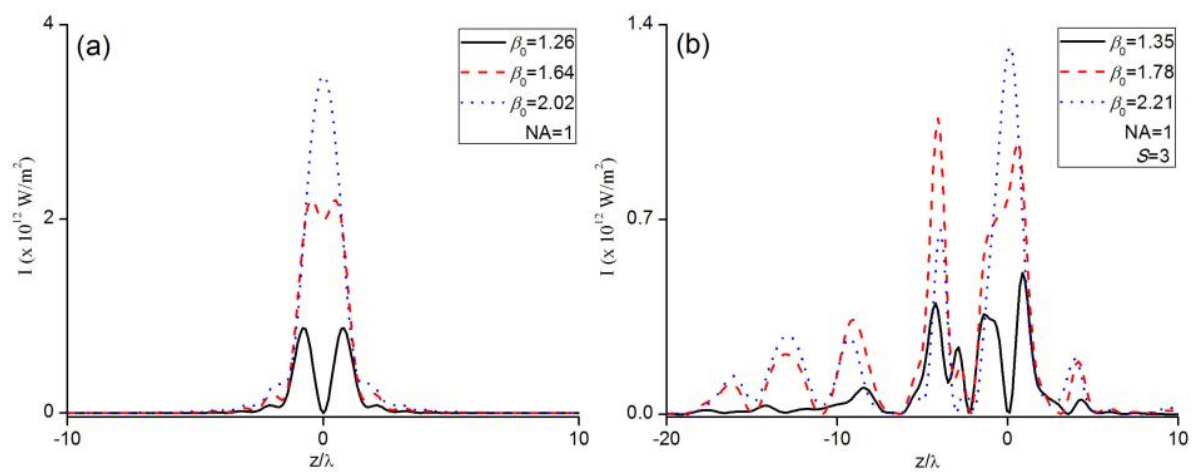

Figure 9. 